\title{
Technè
}

La science au service de l'histoire de l'art et de la préservation des biens culturels

$44 \mid 2016$

Archives de l'humanité : les restes humains patrimonialisés

\section{La momie péruvienne du musée des Confluences : exemple de conservation, de soclage et de mise en exposition d'un élément humain}

The Peruvian mummy in the Musée des Confluences: an example of the conservation, mounting and display of a human element

\section{Marie-Paule Imberti}

\section{OpenEdition} Journals

Édition électronique

URL : http://journals.openedition.org/techne/1169

DOI : 10.4000/techne.1169

ISSN : 2534-5168

\section{Éditeur}

C2RMF

\section{Édition imprimée}

Date de publication : 1 novembre 2016

Pagination : 108-113

ISBN : 978-2-7118-6339-6

ISSN : $1254-7867$

\section{Référence électronique}

Marie-Paule Imberti, «La momie péruvienne du musée des Confluences : exemple de conservation, de soclage et de mise en exposition d'un élément humain », Technè [En ligne], 44 | 2016, mis en ligne le 19 décembre 2019, consulté le 22 juillet 2020. URL : http://journals.openedition.org/techne/1169 ; DOI : https://doi.org/10.4000/techne.1169

\section{(c)}

La revue Technè. La science au service de l'histoire de l'art et de la préservation des biens culturels est mise à disposition selon les termes de la Licence Creative Commons Attribution - Pas d'Utilisation Commerciale - Pas de Modification 4.0 International. 


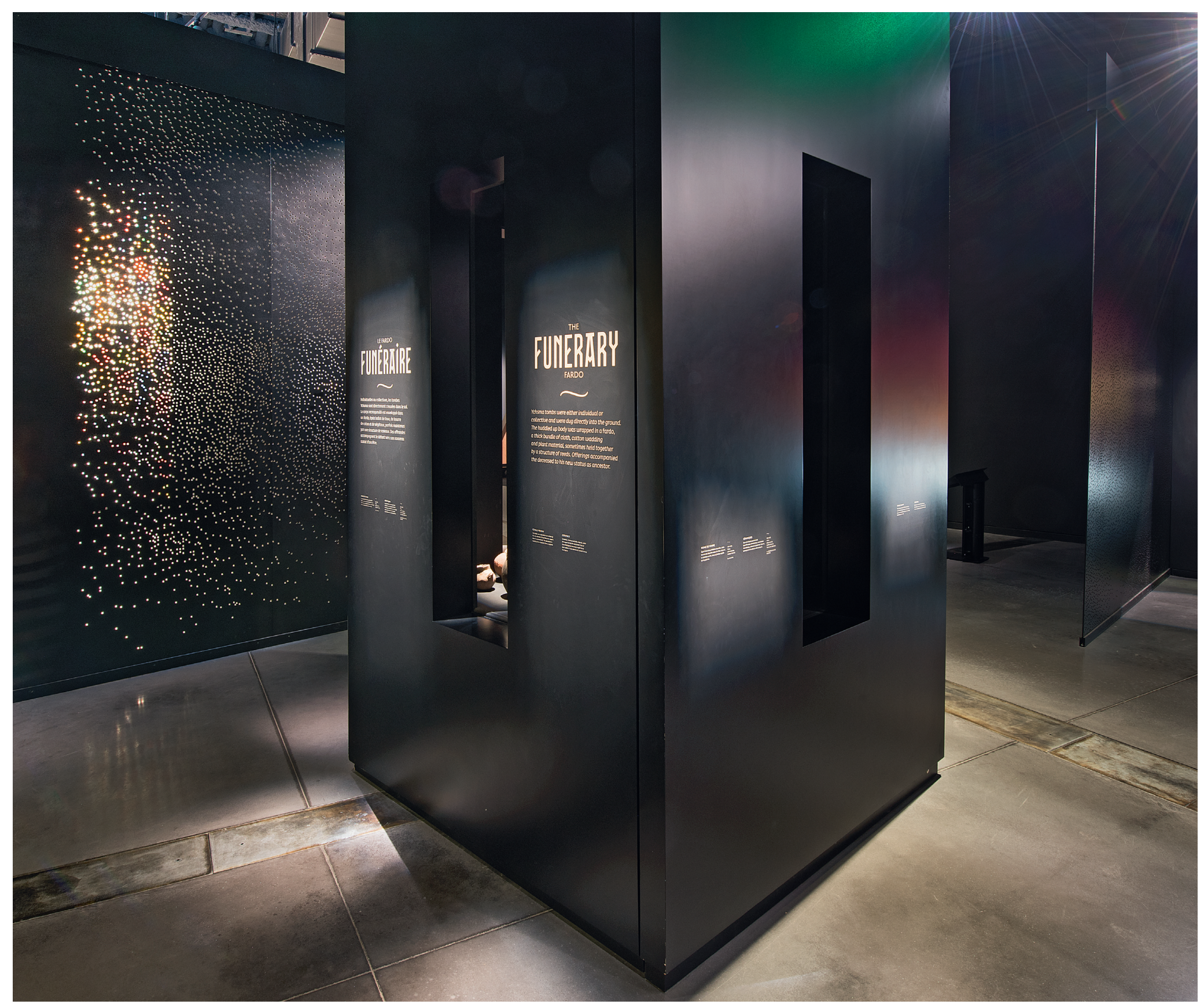

Fig. 1. Mobilier scénographique pour la mise en exposition de la momie. @ B. Stofleth. 
Marie-Paule Imberti

En collaboration

avec Rémi Chauvirey †

et Isabelle George-Aucoin

\section{La momie péruvienne du musée des Confluences : exemple de conservation, de soclage et de mise en exposition d'un élément humain}

Cet article est dédié à la mémoire

de Rémi Chauvirey (1963-2016) (fig. 2).
The Peruvian mummy in the Musée des Confluences: an example of the conservation, mounting and display of a human element
Résumé. Selon un acte de vente en date du 15 décembre 1903, un Lyonnais, Victor François Cotte, travaillant dans l'industrie textile, vend au Muséum d'histoire naturelle de Lyon, deux momies de femmes et divers artéfacts de l'époque précolombienne provenant du Pérou. Plus de cent années plus tard, en décembre 2014, les visiteurs du musée des Confluences, nouvellement ouvert au public, découvrent une de ces deux momies présentée au sein du parcours permanent, dans l'exposition "Éternités, visions de l'au-delà ». Quelles ont été les questionnements et les techniques mises en œuvre pour répondre à la question du soclage d'un corps humain? Et comment ce travail s'est-il inscrit dans une réflexion plus large autour de la conservation et de la mise en exposition d'éléments humains? Conservateur, restaurateur, socleurs, mouleur, chercheurs spécialisés ou encore muséographes et scénographes se sont penchés sur ces délicates questions, mêlant éthique, techniques et expérience humaine.

Mots-clés. Soclage, moulage, momie, élément humain, 3D, empreinte, exposition, conditionnement, éthique, Pérou, musée des Confluences.

\begin{abstract}
According to a deed of sale dated 15 December 1903, a man from Lyon who worked in the textile industry, Victor François Cotte, sold two pre-Columbian female mummies and various artefacts from Peru to the Muséum d'Histoire Naturelle, Lyon. Over one hundred years later, in December 2014, visitors to the Musée des Confluences, recently opened to the public, discovered one of these two mummies displayed in the permanent collection in the exhibition entitled "Eternities, Visions of the Beyond". Which questions were raised and which techniques were used to respond to the idea of mounting a human body on a pedestal? And how did these efforts fit into the broader issue of conserving and exhibiting human elements? Curator, restorer, plinth makers, cast makers, specialized researchers, exhibition and set designers all looked into these delicate questions combining ethics, techniques and human experience.
\end{abstract}

Keywords. Mounting, casting, mummy, human element, 3D, impression, exhibition, conditioning, ethics, Peru, Musée des Confluences.

\section{Introduction}

En 2009, l'archéologue péruvien Leonid Velarde débute une étude documentaire de la collection précolombienne du musée des Confluences en vue d'une mise à niveau des connaissances pour la valorisation de ce fonds. Cette collection, entrée au Muséum d'histoire naturelle principalement à la fin du $\mathrm{XIX}^{\mathrm{e}}$ siècle et au tout début $\mathrm{du} \mathrm{XX}^{\mathrm{e}}$ siècle, se compose majoritairement de différents types de céramiques, de quelques échantillons de textiles, de boîtes à ouvrages, d'une tête postiche, de calebasses gravées, et d'un ensemble d'éléments humains dont les deux momies vendues par Victor François Cotte. La première phase de cette étude, consacrée aux divers mobiliers, permet de dégager une problématique funéraire prédominante. En 2011, une deuxième phase d'étude est lancée sur l'observation anthropologique des momies humaines et des autres éléments humains, crânes et têtes momifiées. Cette nouvelle recherche, confiée à Leonid Velarde et à la bio-anthropologue Sonia Guillén ${ }^{1}$, coïncide alors avec celles menées sur les éléments humains égyptiens du musée des Confluences ${ }^{2}$. Les résultats des diverses observations font émerger une des deux momies plus particulièrement, par ce qu'elle dévoile des pratiques funéraires de son époque. Sonia Guillén décide de la nommer « la tisserande », en regard aux fuseaux et fusaïoles fermement enserrée dans l'une des mains de la momie. Cette dernière, possiblement âgée d'une cinquantaine d'années, a été ensevelie avec son matériel de tisseuse et est très probablement associée à la culture précolombienne Yschma ${ }^{3}$. L'ensemble des recherches confirme ainsi l'importance scientifique et l'intérêt scénographique de ce fonds de collection.

Les éléments humains ne sont pas ici envisagés comme les autres objets de collection. Leur statut est spécifique. Ainsi, ils ne sauraient être manipulés, conservés ou exposés au public

Marie-Paule Imberti, chargée des collections des Amériques, Cercle polaire et exposition, musée des Confluences de Lyon (marie-paule. imberti@museedesconfluences.fr). En collaboration avec Rémi Chauvirey t, socleur, musée des Confluences de Lyon, et Isabelle George-Aucoin, mouleur, musée des Confluences de Lyon (isabelle.george-aucoin@museedesconfluences.fr). 
Rémi Chauvirey débute sa carrière de socleur au sein du musée des Confluences en 2008. Il effectue un nombre conséquent de soclages divers et variés, depuis les fragiles tiges employées pour les objets délicats jusqu'aux structures complexes pour les pièces massives. Rémi Chauvirey était passionné par son métier, il faisait preuve d'un respect absolu de l'objet, et ses interventions se faisaient si discrètes et précises que le socle semblait parfois s'effacer. Cet article, pour lequel nous avions entamé la rédaction sur la partie du soclage, entre le choix technique proposé et son expérience de socleur face à cet "objet » si particulier qu'est le corps momifié, représente aujourd'hui un hommage à la mémoire tant de l'homme qu'il était que du professionnel expérimenté.

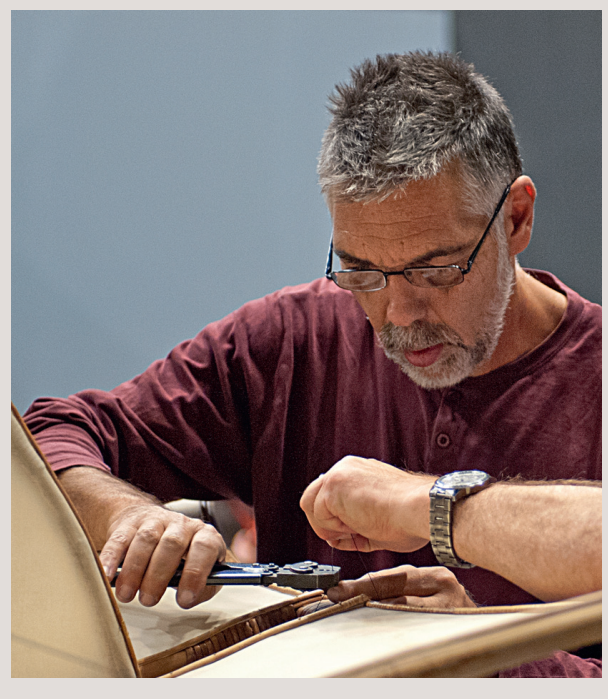

Fig. 2. Rémi Chauvirey (19632016). () Musée des Confluences/ O. Garcin. sans tenir compte de leur humanité, sans préserver la dignité des corps, et sans en respecter l'intégrité. Des pratiques particulières doivent donc être envisagées à tous les niveaux, de la conservation à la présentation au public ${ }^{4}$.

\section{La mise en exposition}

"Éternités, visions de l'au-delà », l'une des expositions du parcours permanent du musée des Confluences, pilotée en interne par Christian Sermet et Carole Millon, propose de poser la question de notre rapport à la mort et d'explorer les différentes manières de l'appréhender, notamment au travers des pratiques funéraires de différentes sociétés anciennes. En 2010, le musée organise une exposition de préfiguration, «Désirs d'éternité ", au Musée gallo-romain de Saint-Romain-en-Gal de Vienne, en vue de tester les éléments prévus pour «Éternités, visions de l'au-delà». Une sépulture de l'Égypte antique et du Caucase constituent alors le support de réflexion sur la question de l'au-delà. Suite à cette exposition, la possibilité d'intégrer un troisième exemple de sépulture se confirme et le choix se porte sur les rites funéraires précolombiens. Fort des résultats d'études, la présentation de la momie péruvienne et de son mobilier funéraire est donc envisagée. Comme pour les deux autres sépultures, il s'agit de trouver le moyen le plus respectueux de présenter le défunt, tout en transmettant les informations nécessaires au visiteur.

Le principe scénographique propose tout d'abord d'appréhender l'espace dédié au rituel funéraire précolombien par une cimaise présentant le texte d'exposition et un audiovisuel diffusant les éléments de compréhension de la thématique. Le visiteur est amené, dans un second temps, à contourner ce premier élément scénographique pour découvrir, au dos, la momie installée au sein d'un mobilier imposant. Celui-ci suggère les dimensions et le volume d'une fosse funéraire précolombienne, avec trois fenêtres de visualisation de type meurtrières qui permettent trois angles de vue différents sur la momie (fig. 1).

Selon le modèle funéraire Yschma, les défunts étaient ensevelis dans des tombes directement creusées dans le sol. Leurs corps recroquevillés étaient complètement dissimulés par un fardo : un épais ballot composé de plusieurs couches de tissu. Ce dernier était quelques fois surmonté d'une tête postiche figurant les traits d'un visage qui redonnait un aspect humain à l'ensemble. Le courrier de vente de 1903 ne donne aucun détail sur l'aspect physique des momies péruviennes lors de leur enregistrement au Muséum de Lyon et les diverses photographies retrouvées dans les archives de l'institution les présentent dépossédées de leur fardo.

Les premières réflexions conduisent très vite à l'idée que ce ballot de textile est primordial pour la bonne compréhension du rituel funéraire par le public, et que la mise en exposition doit pallier ce manque. Par ailleurs, il s'agit également de respecter la pratique traditionnelle de protection corporelle et visuelle du corps de la momie. La reconstitution d'un ballot est alors envisagée grâce à l'expertise de Leonid Velarde, Luisa Diaz Arriola ${ }^{5}$, docteur en archéologie, et Patricia Landa Cragg, conservatrice-restauratrice péruvienne spécialisée en textiles anciens. En s'appuyant sur des éléments documentaires et sur l'expérience de la restauratrice, il s'agit de reproduire un ballot de textile conforme à ceux utilisés à l'époque précolombienne, tout en y opérant une « coupe » pour visualiser la position fotale du défunt. Cette piste est cependant jugée trop complexe à mettre en œuvre et doit être abandonnée au profit d'une proposition plus suggestive. Le dispositif scénographique ${ }^{6}$ s'oriente finalement vers l'utilisation d'un tulle noir. Celui-ci entoure la momie, comme un tube suspendu du haut en bas de la vitrine, laissant transparaitre la position recroquevillée du corps, et un éclairage spécifique vient compléter cette mise en scène. Une ambiance de lumière tamisée permet ainsi à la fois de respecter les conditions de conservation en exposition et de ne pas présenter la momie 
« de pleins feux » aux regards des visiteurs, mais bien de marquer une distance visuelle comme un voile de pudeur face à ce corps humain. Par ailleurs, ce dispositif dissimule la dégradation importante du visage de la momie. Dans un souci de transmission d'informations au public, un focus plus lumineux est principalement porté sur les fuseaux qu'elle tient dans l'une de ses mains, faisant référence à son activité de tisseuse, et des plumes d'oiseau marin dans l'autre main, qui évoque le milieu côtier au sein duquel la momie a évolué. Cet éclairage spécifique procure enfin l'effet d'une lumière pénétrant de la surface à l'intérieur de la sépulture, figurant la découverte des tombes et rappelant l'enfouissement dans le sol pratiqué lors du rituel funéraire.

Ce dispositif propose une posture particulière au visiteur. Le concept scénographique ainsi adopté l'invite à choisir sa propre manière de « rencontrer » la momie. Il est amené à être acteur du contact qu'il souhaite établir avec elle, les réactions pouvant être très variées d'une personne à l'autre. Il lui est donc proposé de faire lui-même, s'il le souhaite, la démarche de s'approcher des fenêtres de visualisation pour une découverte plus « intime » de la momie ou de rester «à distance » tout en distinguant les éléments essentiels que le musée souhaite transmettre, comme la position fœtale du corps ou encore le positionnement traditionnel du matériel funéraire.

L'enjeu de la mise en exposition de la momie est multiple. Au-delà des questions scénographiques, il convient de s'assurer avant tout qu'elle supportera physiquement l'exposition. Il s'agit également de réfléchir au support à mettre en œuvre pour maintenir sa position accroupie au sein de sa vitrine, en gardant à l'esprit les conditions particulières de traitement à appliquer à cet élément humain. Comment ainsi éviter les multiples manipulations, positionner les attaches du soclage sans agresser les parties fragilisées du corps, ou encore, comment le rendre « invisible » et non intrusif.

\section{Études préalables}

En 2012, le musée sollicite l'avis du C2RMF sur la mise en exposition de la momie. Noëlle Timbart, conservatrice auprès de la filière Archéologie et Ethnographie du C2RMF et chargée des Antiquités égyptiennes et orientales, rédige alors un compte-rendu sur cette question après un nouveau constat d'état complet de la momie. Il apparaît effectivement essentiel de s'assurer que l'état physique de la momie peut supporter l'exposition. Dans le sillage des études menées en parallèle sur la collection de momies humaines égyptiennes, nous engageons, grâce à l'aimable autorisation du service de radiologie de l'hôpital Édouard Herriot de Lyon, une campagne de radiographies des momies péruviennes. Le but est à la fois d'enrichir les données scientifiques recueillies jusque-là et d'établir un constat de conservation approfondi en vue de la mise en exposition. Les observations radiographiques traduites par le docteur Samuel Mérigeaud nous confirment que l'intégrité physique de la momie permet d'envisager sa mise en exposition.

\section{Le soclage, des essais à la réalisation}

La question de la réalisation du support matériel de la momie, ou soclage, représente un enjeu central de la mise en exposition. Comment obtenir un résultat satisfaisant d'un point de vue technique et éthique ? Il est nécessaire de s'entourer d'une équipe de spécialistes. Laure Cadot, spécialisée dans la restauration des éléments humains, a apporté son assistance au musée pour la réalisation du soclage de la momie auprès des équipes internes. Elle a travaillé en collaboration étroite avec Rémi Chauvirey, socleur, et Isabelle George-Aucoin, mouleur. Les premières rencontres s'effectuent également avec Leonid Velarde.

Le rapport de Laure Cadot ${ }^{7}$, réalisé à la suite d'observations de la momie au début de l'année 2013, fournit les précisions nécessaires à la poursuite du travail. La restauratrice confirme ainsi les conclusions relevées préalablement par le docteur Samuel Mérigeaud, sur le fait que la momie ne présente pas de disjonction des connexions anatomiques ou de parties mobiles risquant de se désolidariser. La présentation en position assise semble donc possible. Néanmoins, un certain nombre de points sont à prendre en compte, comme la dégradation avancée de l'os de la mandibule entraînant un risque important de déchaussement des dents, la grande fragilité des cheveux qui se désolidarisent du crâne ou encore la peau devenue extrêmement sèche et sujette aux craquellements et aux déchirures. De plus, « la peau est (...) lacunaire sur les côtés de l'abdomen et de la cage thoracique sur le côté dextre ainsi qu'au niveau du bassin sur les zones n'étant pas directement supportées par l'os sous-jacent ». Ces espaces de peau non supportées par l'os ont ainsi subi des enfoncements à l'origine de pertes de matière engendrant une fragilité et un aspect visuel particulier qu'il convient de prendre en compte tant pour le choix de placement du soclage sur le corps que pour les parties du corps qui ne seront pas montrées. Par ailleurs, le rapport comporte des schémas de préconisation pour le soclage (fig. 3) et de présentation muséographique de la momie avec un ballot reconstitué.

Les contraintes de soclage sont multiples et présentent des difficultés complexes. Étant donné que la momie doit être présentée assise, le socle se doit d'épouser la forme de son dos en partant d'une platine au sol et en remontant le long de la colonne pour venir « crocheter » les clavicules de manière à assurer la stabilité de l'ensemble. Les observations fournies par Laure Cadot rappellent que les manipulations sont à éviter et qu'il est bien évidemment impossible de coucher la momie «sur le ventre » pour réaliser le support dorsal. Après concertation, le constat d'un soclage à réaliser à partir d'un fac-similé du dos de la momie apparaît comme évident et il s'agit alors d'entrevoir les possibilités de la réalisation d'une telle pièce.

La technique du moulage est tout d'abord envisagée. Rémi Chauvirey sculpte dans un premier temps, une réplique 


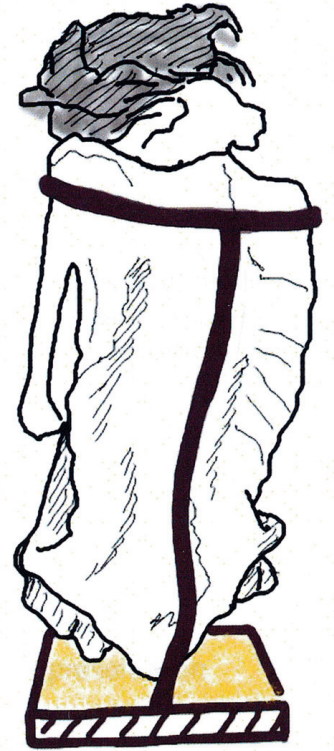

Fig. 3. Schéma préparatoire du principe de soclage retenu pour la mise en exposition. (c) L. Cadot.

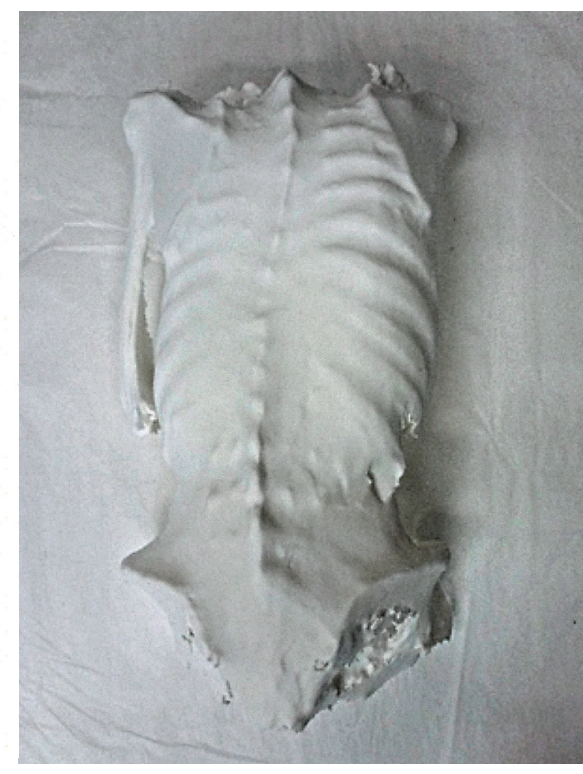

Fig. 4. Impression en 3D du dos de la momie. (C) Musée des Confluences/R. Chauvirey.

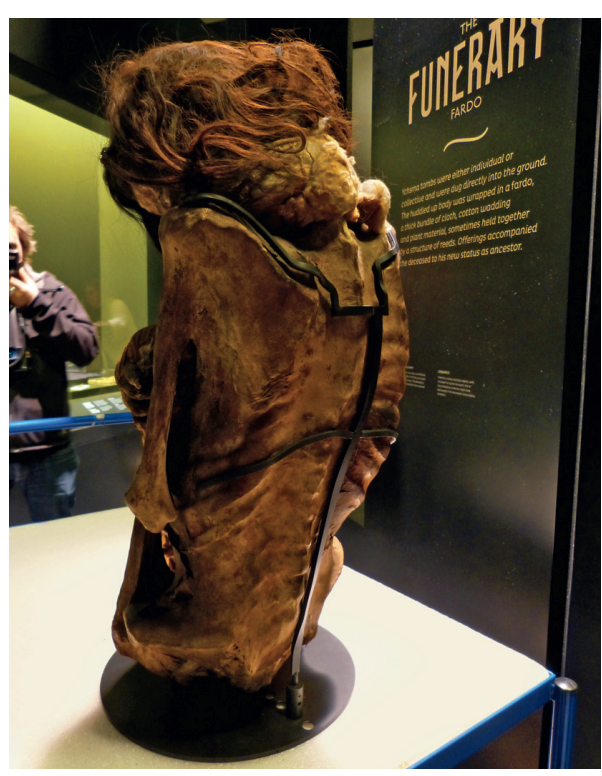

Fig. 5. Ajustement du soclage au dos de la momie avant la mise en vitrine. (c) L. Cadot. du dos de la momie en mousse polyéthylène. Isabelle GeorgeAucoin, accompagnée de Laure Cadot, tentent alors un premier essai de prise d'empreinte à partir de ce fac-similé, en utilisant un plâtre armé de «filasse ", matériau en fibre végétale. L'équipe se confronte rapidement à plusieurs difficultés. Le poids trop volumineux du matériau utilisé pour la réalisation du moule, le fort dégagement thermique occasionné et les dommages accidentels pouvant survenir en cas de contredépouille représentent des risques jugés trop importants. Cette technique ne peut donc pas être adaptée à la momie.

\section{La technologie 3D au service de l'élément humain}

Face à ces premières constatations, Rémi Chauvirey propose de tenter une autre technique. Celle-ci pourrait résoudre la question de la manipulation de la momie tout en permettant d'obtenir un support de fabrication pour le soclage dans les meilleures conditions, tant pour le socleur que par respect pour le corps de « la tisserande».

Le musée des Confluences se dote en 2012 d'un scanner portable 3D Artec ${ }^{\circledR 8}$, dans le but d'effectuer la modélisation et la préfiguration, avant l'ouverture de l'institution en décembre 2014, de certaines vitrines de son parcours permanent. Cette technique basée sur la capture 3D permet également d'obtenir des tirages en résine d'objets en petite série pouvant servir à divers usages, supports de médiation ou répliques proposées «à toucher " aux visiteurs. Le scanner permet donc la numérisation en volume d'objets de taille variable et de textures multiples sans avoir à effectuer de manipulations trop intrusives sur les objets de collection particulièrement délicats. Cette technologie a donc un avantage certain pour répondre au problème de manipulation liée à la momie. Le but consiste à obtenir un support identique au dos de celle-ci par l'impression d'un fac-similé en résine. Ainsi, le socleur peut envisager son support au plus près des courbes et des contraintes physiques du corps reproduit sur le fac-similé pour proposer un soclage assurant un maintien optimal et non contraignant pour la mise en exposition.

Techniquement, l'appareil «saisit» une multitude de « photos » qui constituent « un fichier» de scans. Pour obtenir une image complète, l'appareil est déplacé autour de la momie pour en saisir toutes les faces. Les manipulations sont réduites à leur minimum et l'opération peut s'effectuer en une seule fois sans avoir besoin de solliciter le corps à plusieurs reprises. L'impression en 3D est confiée à l'antenne parisienne de la société Crésillas ${ }^{9}$, choisie par le musée pour sa capacité à effectuer des tirages de grande dimension, en une seule pièce, (fig. 4) répondant au souci de précision exigé pour la création du support.

Lors du montage final, la fabrication du soclage est déléguée à l'entreprise Aïnu ${ }^{10}$, un des prestataires engagés pour l'ouverture de l'institution. Louis-Albin de Chavagnac, chef de projet au sein de cette entreprise, adapte sur place le soclage pensé par Rémi Chauvirey et finalise sa pose au dos de la momie. L'efficacité de cette solution est remarquable. Le socle agit comme un « habit de peau » (fig. 5), discret, sur mesure, en respectant la morphologie du dos et sans intrusion à l'intérieur du corps de la momie, comme ont pu l'être des systèmes antérieurs de soclage.

\section{Conclusion}

«La question de la préservation, de l'exposition, mais aussi du conditionnement des restes humains fait l'objet d'une réflexion permanente au sein d'un musée qui a inscrit le respect de la personne humaine dans ses valeurs 
fondamentales ${ }^{11}$. » Cette notion de considération particulière du corps de la momie a donc constitué le fil conducteur des interventions, de sa conservation à son exposition. La mise en scène spécifique au sein du parcours permanent, la réalisation d'un soclage «sur mesure » et l'adaptation des conditionnements démontrent aujourd'hui toute leur efficacité et conforte l'équipe dans l'idée que les réflexions menées en commun ont abouti aux résultats escomptés. Les éléments humains péruviens du musée des Confluences restent les gardiens d'un passé précolombien. Ces derniers n'ont pas encore livré tous leurs secrets, tant en terme de transmission des connaissances de la société au sein de laquelle ils ont évolué que sur les personnes humaines qu'ils ont été et dont le musée se doit aujourd'hui de conserver les corps.

\section{Un conditionnement adapté}

En 2011, les études menées sur les momies égyptiennes et sur les momies, crânes et têtes momifiées péruviennes ont donné lieu à une campagne générale d'amélioration des conditions de conservation des éléments humains du musée des Confluences. À partir des compétences en interne, des expériences partagées et des discutions avec des spécialistes des momies et de la conservation, de nouveaux conditionnements voient le jour (fig. 6). Jusque-là simplement entreposées sur les étagères en réserve, les momies sont alors transférées dans des boîtes en polypropylène cannelé, à 4 pans rabattables et munies de poignées facilitant la manipulation et les transports. Les parois internes des boîtes sont capitonnées de mousse recouverte de Tyvek ${ }^{\circledR}$. À l'intérieur, la momie repose sur un plateau amovible qui permet de la transférer de sa boîte à une

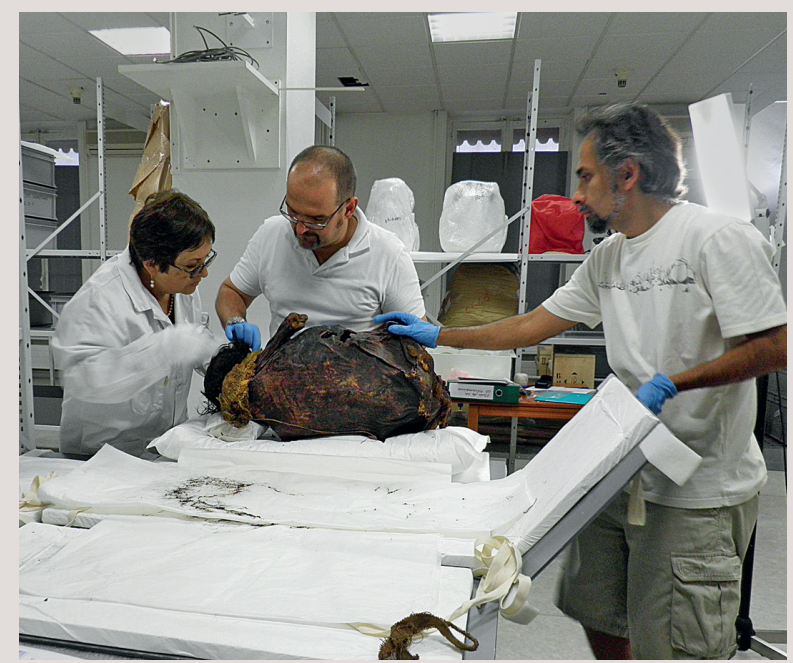

Fig. 6. L'exemple d'une momie transférée de son conditionnement adapté, pour l'étude menée par Sonia Guillén et Leonid Velarde. ○ Musée des Confluences/M.-P. Imberti.

table de consultation par exemple sans avoir à la toucher. Les momies péruviennes reposent sur un coussin en toile de coton remplis de microbilles épousant parfaitement les courbes de leur corps recroquevillé en position fotale et évitant ainsi des tensions dangereuses pour leur intégrité physique. Ce type de conditionnement, même s'il reste toujours perfectible, propose aujourd'hui une conservation optimale des momies. Ils ont été pensés et élaborés pour répondre à la fois aux exigences de conservation préventive et aux questions d'éthique liées aux éléments humains. Ainsi, la dignité des corps est préservée et les détériorations qui auraient pu être engendrées par les nombreuses manipulations effectuées au cours des différentes étapes d'études et de recherches se sont vues nettement diminuées ${ }^{12}$.

\section{Notes}

1. Sonia Guillén était, à l'époque de l'étude, directeur du museo Leymebamba, Chachapoyas, Amazonas, depuis 1998. Elle est également directeur du Centro Mallqui/ The bioanthropology fondation depuis 1993

2. Madrigal et Emmons, 2012.

3. Imberti et Velarde, 2012, p. 34

4. Novljanin Grignard, 2012.

5. Diaz Arriola, 2012.

6. Agence Klapisch-Claisse (Marianne Klapisch et Mitia Claisse) et Étienne Le François, Paris.

7. Rapport d'étude et de préconisation de conservation pour la présentation de la momie péruvienne inv. 81000106 dite "la tisserande", 2013.

8. Scanner portable permettant d'obtenir la numérisation en 3D d'objets à géométrie complexe et à texture riche, en haute résolution.

9. Cette société lyonnaise est spécialisée dans l'impression 3D depuis 1993. Le tirage a ainsi été produit par le procédé de stéréolithographie, ou "écriture en relief », capable de réaliser n'importe quelle géométrie issue d'une modélisation en 3D, quel que soit son degré de complexité. https://www.cresilas.fr

10. Société de soclage, production et conservation, Paris.

11. Bruno Jacomy, directeur scientifique du musée des Confluences (2005-juillet 2016.

12. Chareyron, 2012, p. 59.

\section{Bibliographie}

Chareyron N., 2012, «Conservation, manipulation et conditionnement des éléments humains, l'expérience du musée des Confluences ", Cahier du musée des Confluences. Études scientifiques 3, p. 57-64.

Imberti M.-P., Velarde L., 2012, « Aperçu de l'étude en cours sur les momies et les crânes humains péruviens de la collection précolombienne du musée des Confluences ", Cahiers du musée des Confluences, Études scientifiques 3 , p. 33-38.

Madrigal K., Emmons D., 2012, « Dernières études sur les momies égyptiennes du musée des Confluences ", Cahiers du musée des Confluences. Études scientifiques 3, p. 29-32.

Diaz Arriola L., 2012, « Analyse d'une tombe Yschma récente », étude documentaire commandée pour le musée des Confluences.

Revue collective, 2012, «Les restes humains au musée des Confluences. Dossier thématique ", Cahiers du musée des Confluences. Etudes scientifiques 3, 84 p.

Novljanin Grignard I., 2012, «Les musées et leurs restes humains : différents regards et nouveaux enjeux ", Cahiers du musée des Confluences. Études scientifiques 3, p. 11 à 16. 